\title{
Cardiac Teratogenicity of Dichloroethylene in a Chick Model
}

\author{
STANLEY J. GOLDBERG, BRENDA V. DAWSON, PAULA D. JOHNSON, H. EUGENE HOYME, \\ AND JUDITH B. ULREICH \\ Departments of Pediatrics [S.J.G., H.E.H.], Internal Medicine [B.V.D., P.D.J.], and Surgery [J.B.U.], University \\ Heart Center, Childrens Research Center, University of Arizona, Tucson, Arizona 85724
}

\begin{abstract}
Trichloroethylene (TCE) and dichloroethylene (DCE) are related halogenated aliphatic hydrocarbon industrial solvents that are frequently found as drinking water contaminants. TCE has been implicated as a cardiac teratogen in an epidemiologic study and in a chick model. The purpose of this study was to determine whether DCE was also a cardiac teratogen in the chick embryo. Fertilized White Leghorn chick eggs $(n=418)$ were inoculated just above the embryo with $30 \mu \mathrm{L}$ of a test solution on d 3 of incubation. Two control groups were studied: normal saline $(n=96)$ and the diluent for the DCE, miner?' oil $(n=$ 108). DCE was studied at three doses: 5, 20, and $25 \mu \mathrm{M}$ $(n=76,62$, and 76, respectively). Eggs were coded with a seven-digit number to mask identity. Chicks were terminated on d 18 of incubation, and, after external inspection, hearts and great vessels were dissected macroscopically according to a detailed protocol. Abnormal hearts were reviewed and the diagnosis was agreed upon by three investigators before decoding the seven-digit number and photographing the abnormality. Some embryo death and subsequent tissue autolysis occurred in all groups, but, compared to controls, it was not significantly greater in the treatment group. However, combining all controls and all experimentals, significantly more $(p=0.02)$ embryonic death occurred in the experimental group. Noncardiac anomalies occurred in 17 embryos and were highest in the saline (four), $5 \mu \mathrm{M}$ (four), and $20 \mu \mathrm{M}$ (seven) DCE groups. Cardiac and great vessel anomalies occurred in $4 \%$ of saline controls, $4 \%$ of mineral oil controls, $17 \%$ of the DCE 5 $\mu \mathrm{M}$ group $(p<0.05), 19 \%$ of the DCE $20 \mu \mathrm{M}$ group ( $p$ $<0.05)$, and $2 \%$ of the DCE $25 \mu \mathrm{M}$ group $(p=0.1)$. Cardiac anomalies included atrial and ventricular septal defects, malformations of all valves, and great vessel abnormalities. Results suggest that DCE is a more potent cardiac teratogen than a general teratogen in the chick. Results for DCE, including the marked decrease in cardiac teratogenicity between 20 and $25 \mu \mathrm{M}$ treated groups, are almost identical to results found for TCE, which suggests that the cardiac teratogenic effect may be exerted through a common mechanism, possibly a common or similar metabolite. (Pediatr Res 32: 23-26, 1992)
\end{abstract}

\section{Abbreviations}

DCE, dichloroethylene

TCE, trichloroethylene

Received May 7, 1991; accepted February 6, 1992

Correspondence and reprint requests: Stanley J. Goldberg, M.D., Department of Pediatrics (Cardiology), University of Arizona College of Medicine, $1501 \mathrm{~N}$. Campbell Ave., Tucson, AZ 85724

Supported by The University of Arizona BRSG, Arizona Affiliate of the American Heart Association, the Arizona Disease Control Research Commission, and NIEHS Superfund project 1P42ES4940.
Vinylidene chloride (1,1-dichloroethylene, DCE) is used as a solvent and in the industrial process of making plastics. DCE and related compounds are also common water supply contaminants (1). Although information is available regarding the acute toxicity of DCE $(2,3)$, less is known of its general teratogenicity or specific cardiac teratogenesis. One prior study suggested that general teratogenicity was limited to minor skeletal abnormalities (3). Although the authors of the prior study indicated that a minimal cardiac examination was performed, no defects were reported.

Two prior epidemiologic studies reported an association of halogenated hydrocarbons with increased major cardiac malformations in children born to mothers who lived in areas of water contamination. The California Health Department found both trichloroethane and DCE in the water supply in an area of Santa Clara County. A greater than expected number of major cardiac congenital anomalies were found in children born to mothers who lived in the area (4). TCE and DCE entered the groundwater in an area of Tucson, Arizona during the $1950 \mathrm{~s}$, contaminating numerous wells from which area residents derived their water supply. Water contamination with TCE and DCE was detected initially in 1981 and affected wells were later closed. University of Arizona pediatric cardiologists had noticed since 1973 that many of their patients with congenital heart defects lived in the area served by the contaminated wells and a subsequent epidemiologic study revealed an approximately 3 -fold relative incidence of congenital cardiac disease as compared to other areas. The increased incidence disappeared after well closure (5). These studies prompted investigation of the cardiac teratogenesis of the contaminating halogenated aliphatic hydrocarbons.

Subsequently, TCE was studied in an avian model during early embryogenesis (6). Data from this study confirmed TCE as an avian teratogen with significantly increased numbers of malformations and embryonic death in treated groups compared with controls. Specific cardiac teratogenicity of TCE in the avian model was also examined (6). TCE-treated embryos had more than 3 times as many congenital heart deformities compared to control groups, and TCE was established as a cardiac teratogen in an avian model.

The present study was designed to determine whether DCE causes cardiac teratogenicity in a chick model when administered during early organogenesis.

\section{MATERIALS AND METHODS}

White Leghorn chick eggs were collected within $24 \mathrm{~h}$ after they were laid and then refrigerated at $42^{\circ} \mathrm{F}$ for no longer than $9 \mathrm{~d}$. Eggs were allowed to equilibrate to room temperature for 6-8 h and then placed in a controlled humidity $(72 \%)$ and temperature $\left(100^{\circ} \mathrm{F}\right)$ automatic incubator. On the afternoon of $\mathrm{d} 3$ of incubation, eggs were candled to locate the embryo. The site was marked on the shell, and the area around the mark was cleaned with $70 \%$ alcohol. A Dremmil drill was used to make a $1-\mathrm{mm}$ hole in the shell approximately $6 \mathrm{~mm}$ above the embryo. Care 
Table 1. Embryo abnormalities

\begin{tabular}{|c|c|c|c|c|c|c|}
\hline & Saline & $\begin{array}{c}\text { Mineral } \\
\text { oil } \\
\end{array}$ & $5 \mu \mathrm{M} \mathrm{DCE}$ & $20 \mu \mathrm{M}$ DCE & $25 \mu \mathrm{M}$ DCE & Total \\
\hline Eggs treated & 96 & 108 & 76 & 62 & 76 & 418 \\
\hline Embryo death & 9 & 13 & 16 & 10 & 16 & 64 \\
\hline External abnormalities & 4 & 0 & $4^{*}$ & $7^{*}$ & 2 & 17 \\
\hline Hearts dissected & 86 & 95 & 60 & 52 & 58 & 351 \\
\hline Normal hearts & 83 & 91 & 50 & 42 & 57 & 323 \\
\hline Abnormal hearts & 3 & 4 & $10 * \dagger$ & $10^{*} \dagger$ & 1 & 28 \\
\hline$\%$ Abnormal hearts & 4 & 4 & $17 * \dagger$ & $19 * \dagger$ & 2 & 8 \\
\hline
\end{tabular}

* Significantly different from mineral oil control $(p<0.02)$.

$\uparrow$ Significantly different from saline control $(p<0.02)$.

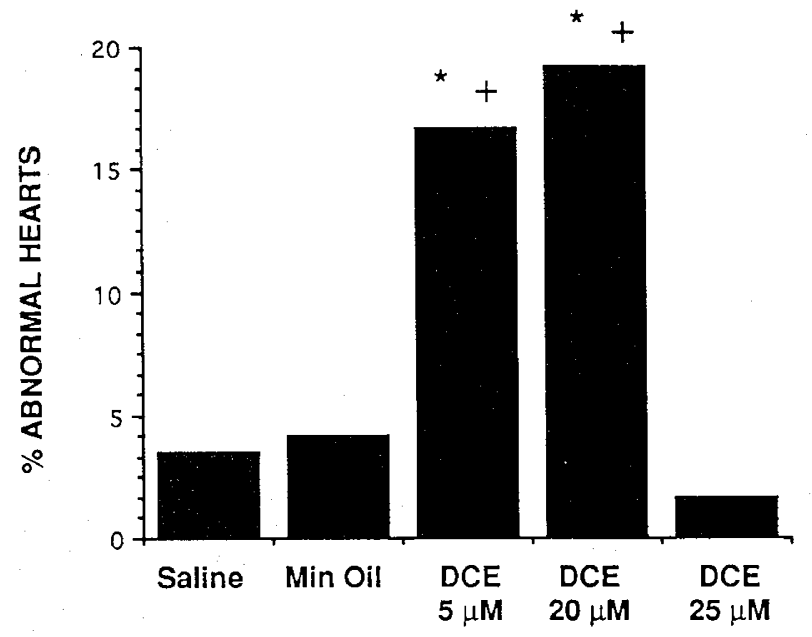

SOLUTIONS ADMINISTERED $(\mu \mathrm{l})$

Fig. 1. Percentage of abnormal hearts. Each bar on the horizontal axis represents a group of embryos, with specific treatments indicated below the horizontal axis. The vertical axis indicates the percentage of abnormal hearts. *, Significantly different from saline control, $p<0.05$; + , significantly different from mineral oil control, $p<0.05$.

was taken not to penetrate the membrane. A sterile $100-\mu \mathrm{L}$ Hamilton syringe was used to inject, just above the embryo, 30 $\mu \mathrm{L}$ of one of the five following solutions: $0.9 \% \mathrm{NaCl}$, mineral oil, or $\operatorname{DCE}(5,20$, or $25 \mu \mathrm{M})$ dissolved in mineral oil. After injection, the hole was sealed with a small piece of transparent tape. The study was conducted in such a manner that investigators were not aware of the treatment. Vials from which the solutions were drawn were coded with numbers, and each egg was coded with a seven-digit number that identified the injected solution and allowed unique identification of each embryo. On d 18, embryos were harvested. Some embryos were dead and autolyzed; development was arrested in most at about stage 26 as judged by eye and limb bud formation (7). Living embryos were terminated and an external examination was performed on each by a veterinarian. Then, a "V" shaped incision was made from the keel to the base of each wing and ribs were drawn cranially to expose the thoracic cavity. The pericardium was gently removed and the heart was inspected (in situ) to determine cardiac location and great vessel morphology. The apex of the heart was brought cranially and the caudal vena cava was incised. Great vessels and cranial vena cava were then incised as distal to the heart as possible and the heart was removed and flushed with $2 \%$ gluteraldehyde solution via an apically placed 27 -gauge needle. It was not possible to remove the autolyzed hearts in the dead embryos. After fixing in gluteraldehyde for $24 \mathrm{~h}$, hearts were transferred to $0.1 \mathrm{M}$ phosphate buffer, $\mathrm{pH} 7.0$, for storage.

Hearts were dissected under a Nikon (Garden City, NY) SMZ$2 \mathrm{~T}$ light microscope equipped with a television monitor. First, the right atrial wall was removed. If complete visualization of the atrial septum was not achieved, the left atrial wall was also removed. Next, the aortic and pulmonary vessels were observed for location, branching, and size. Then, the great vessels were excised at their bases and the remaining atrial wall and septum were removed to permit direct examination of all four cardiac valves. The left ventricular free wall was removed by making a triangular incision through the left atrioventricular valve to allow visualization of the ventricular septum from the left. A similar triangular incision was made from the right atrioventricular valve to the apex of the heart and extended up to the pulmonary valve, thus exposing the entire outflow tract and the interventricular septum from the right. All abnormalities required agreement by three investigators. Significance of data was tested by the $\chi^{2}$ method with continuity correction.

\section{RESULTS}

A total of 418 embryos were studied, and Table 1 summarizes the results. There was disagreement over only one specimen. Results regarding that specimen were eliminated.

Embryo death. Of the 418 embryos, 64 (15\%) exhibited embryonic death (Table 1). Hearts could not be studied in these embryos because of autolysis. Embryo death occurred in $9 \%$ of eggs injected with $0.9 \% \mathrm{NaCl}$ and in $12 \%$ of those injected with mineral oil. The difference in embryo death between the two control groups was not significant. Percentage of embryo death for the DCE groups was as follows: $5 \mu \mathrm{M}, 21 \%$; $20 \mu \mathrm{M}, 16 \%$; and $25 \mu \mathrm{M}, 21 \% . \chi^{2}$ testing revealed that the results of no group were significantly different from controls. A comparison of all controls and all experimentals demonstrated that significantly more $(p=0.03)$ embryonic death occurred in experimentals than controls.

External abnormalities. Four percent of the embryos (17 of

Table 2. Cardiac abnormalities in controls and DCE-treated chicks

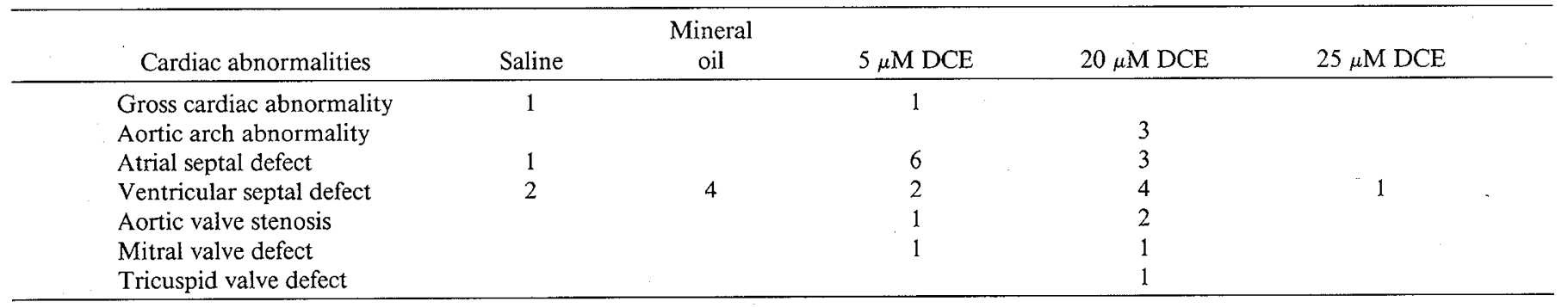




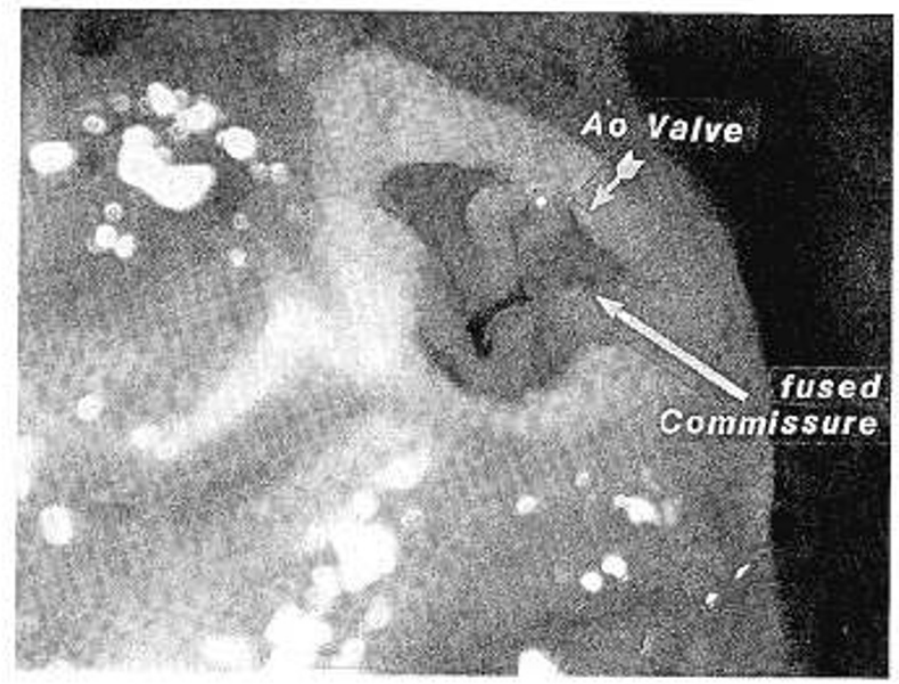

Fig. 2. Example of aortic valvular stenosis: Aortic stenosis created by fusion of two cusps. The fusion is marked with an arrow. Ao, aortic.

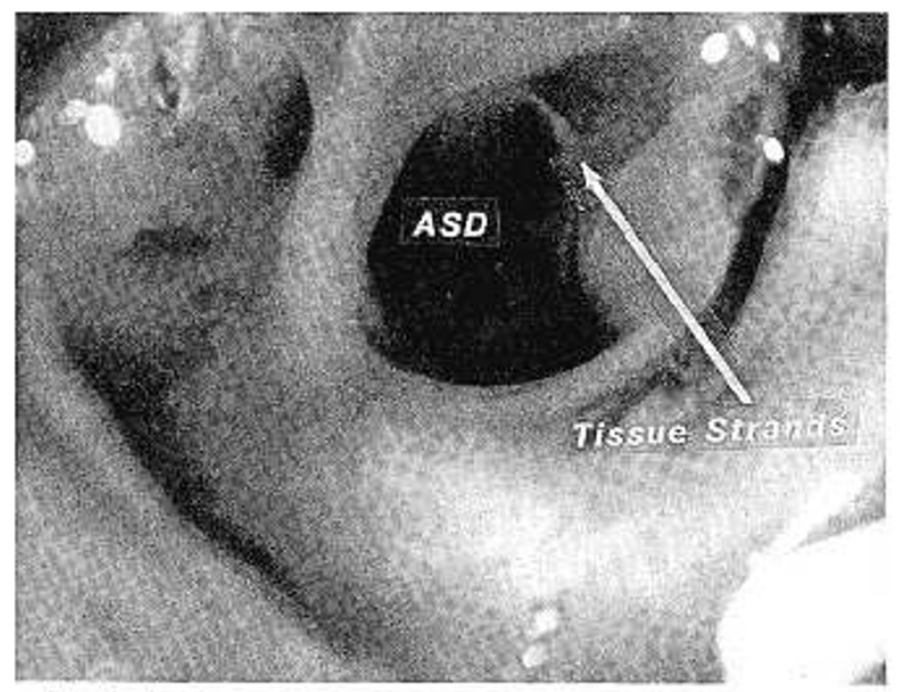

Fig. 3. Exampie of atrial septal defect $(A S D)$. Note that several strands of tissue cross the defect, but no tissue is available to close the defect.

418) exhibited external abnormalities (Table 1), including anophthalmus, ablepharia, encephalocele, thoracogastroschisis, maxillary deviation, and contracted tendons. No external abnormalities were found in the mineral oil control group $(n=108)$, but four occurred in the saline control group $(n=96) . \chi^{2}$ testing indicated that significantly more $(p<0.05)$ external abnormalities occurred in the $5-\mu \mathrm{M}(5 \%)$ and $20-\mu \mathrm{M}(11 \%)$ DCE groups than in the mineral oil group, but a comparison of the number of external anomalies for the saline control group and the numbers in the DCE groups showed no significant difference. External abnormalities for the $25-\mu \mathrm{M}$ DCE group (3\%) were not significantly different when compared to control.

Cardiac abnormalities. A total of 28 abnormal hearts were found, seven in controls and 21 in experimental embryos (Table 1 ). The number of abnormal hearts ( 28 of 351 ) was significantly higher $(p<0.03)$ than the number of embryos with noncardiac anomalies (17 of 418). Abnormal hearts occurred in $4 \%$ of the saline group and $4 \%$ of the mineral oil group (no significant difference). The 5- and 20- $\mu \mathrm{M}$ DCE-treated groups had 17 and $19 \%$ abnormal hearts, respectively, and results of both groups were significantly different from results of control groups $(p \leq$ 0.05 ) (Table 1, Fig. 1). The 25- $\mu \mathrm{M}$ DCE-treated group had only $2 \%$ abnormal hearts, and this was not significantly different from

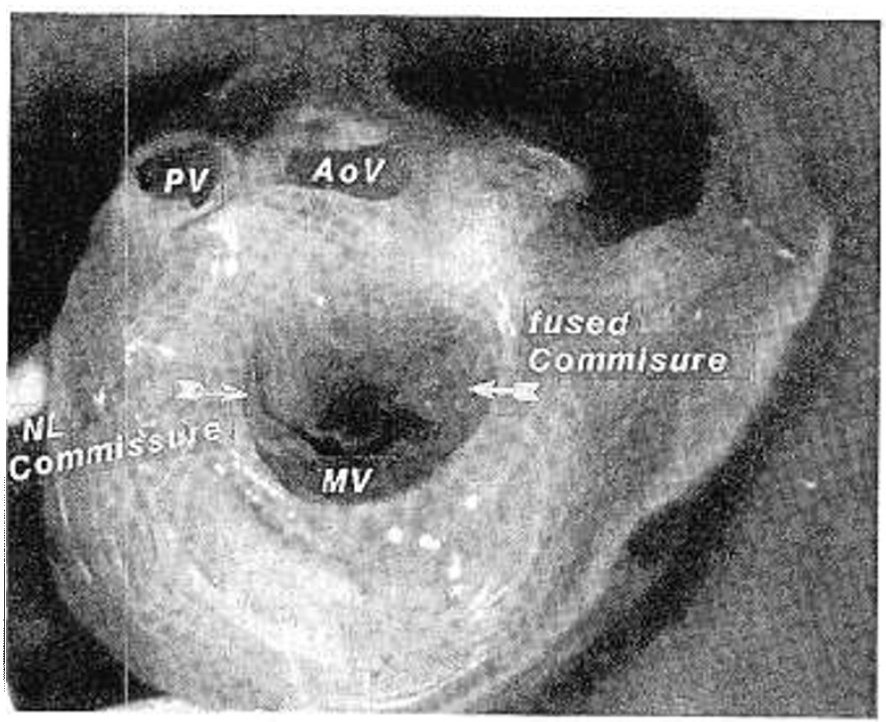

Fig. 4. Example of mitral stenosis. Fusion (arrow) of the anterior and posterior mitral valve leaflets. Note the normal separation of cusps opposite the fusion. View is from the posterior aspect, with the aortic valve viewed directly anteriorly. $A O V$, aortic valve; $N L$, normal; $M V$, mitral valve; and $P V$, pulmonary valve.

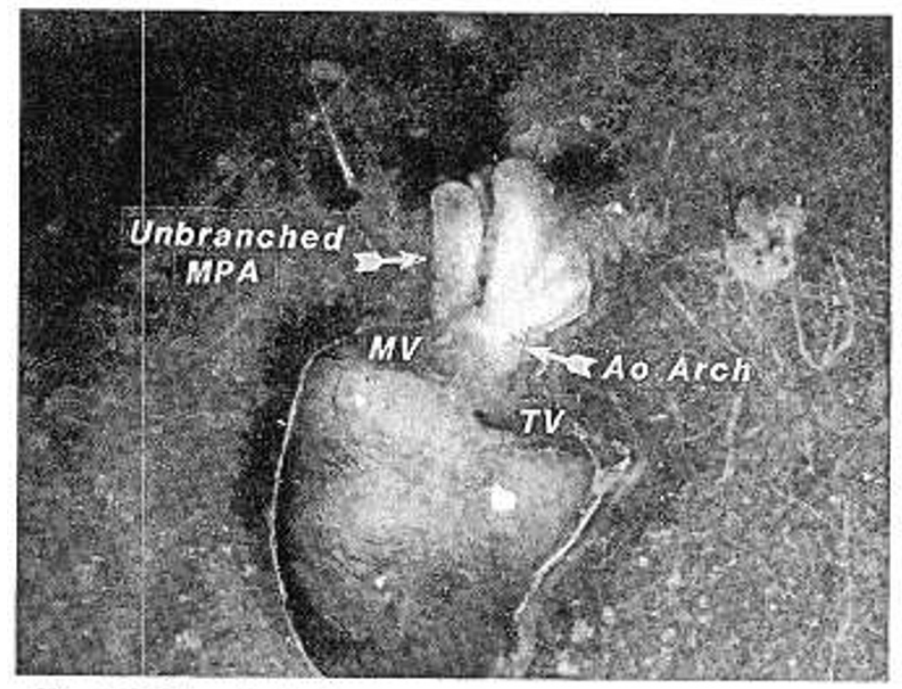

Fig. 5. Unbranched puimonary artery. Faulure of division of the main pulmonary artery into a right and left branch. The heart is viewed from the posterior aspect. The aortic arch is normal. Tricuspid and mitral valves are identified. $A O$, aortic; $M P A$, main pulmonary artery; $M V$, mitral valve; and $T V$, tricuspid valve.

controls. The classification of heart abnormalities is shown in Table 2 (examples of cardiac defects are shown in Figs. 2-5.) Ventricular septal defect types are subclassified in Table 3. Most controls (five of six) had ventricular septal defects localized to the subaortic region, whereas defects in the $5-\mu \mathrm{M}$ and particularly the $20-\mu \mathrm{M}$ group were distributed to other locations as well as the subaortic area. Atrioventricular valve abnormalities included two hypoplastic mitral valves, one of which was associated with a large tricuspid valve and a ventricular septal defect. Semilunar valve abnormalities included stenosis due to cusp adhesions in two and a hypoplastic valve ring with hypoplastic leaflets in another. Great vessel abnormalities included abnormal branching, and one had a hypoplastic ascending aorta.

\section{DISCUSSION}

The most important findings of this study are that 1) administration of DCE on the $3 \mathrm{rd} \mathrm{d}$ of incubation produced a signifi- 
Table 3. VSD location according to treatment

\begin{tabular}{|c|c|c|c|c|c|}
\hline VSD location & $\begin{array}{c}\text { Mineral } \\
\text { oil }\end{array}$ & $0.9 \mathrm{NaCL}$ & $5 \mu \mathrm{M} \mathrm{DCE}$ & $20 \mu \mathrm{M} \mathrm{DCE}$ & $25 \mu \mathrm{M} \mathrm{DCE}$ \\
\hline Subpulmonary & 1 & & & 1 & \\
\hline Subaortic & 3 & 2 & 1 & 1 & 1 \\
\hline Malalignment & & & & 1 & \\
\hline Doubly committed & & & & 1 & \\
\hline
\end{tabular}

cantly increased percentage of embryos with cardiac abnormalities as compared to controls; 2) the number of cardiac anomalies was significantly greater than the number of noncardiac abnormalities; and 3) the greatest frequency of cardiac abnormalities occurred at doses of 5 and $20 \mu \mathrm{M}$ DCE, but the frequency of cardiac abnormalities was not increased above control levels for the $25-\mu \mathrm{M}$ dose.

Chick development occurs within a $21-\mathrm{d}$ incubational period. Major cardiogenic events span d 3-8, and include septation of the truncus arteriosus, atria, and ventricles, foraminal formation in the atrial and ventricular septa, and cushion and valve formation. At the end of $\mathrm{d} 8$, cardiac formation is essentially complete, but minor remodeling continues through $\mathrm{d} 21$. By $\mathrm{d}$ 21 , remodeling of atrial and ventricular septa is complete, which results in subsequent closure of the foramina and establishment of a mature, adult-like heart.

Our results for DCE are similar to those reported previously by Loeber et al. (6) for TCE. They used similar methodology to study TCE and found a peak percentage of cardiac abnormalities at $20 \mu \mathrm{M}(21 \%)$ and fewer at $25 \mu \mathrm{M}(3 \%)$. In the present study, no dose response was found and, as in the Loeber study, no particular lesion predominated, although ventricular septal defects were the most common. The TCE study and the epidemiologic study showed no predilection toward any special lesion. In the present study, the location and type of ventricular septal defects of the controls (five of six subaortic) were somewhat different from those of treated embryos (three of seven). Loeber et al. (6) did not evaluate ventricular septal defect type. The percentage of cardiac abnormalities in the Loeber control group was not significantly different from that reported here. The combined data of Loeber et al. and the present DCE study suggest that the two compounds, TCE and DCE, exert a similar influence on cardiac development or share a common or closely related metabolite that affects cardiac development.

One prior study (3) by another group of the possible teratogenic effect of DCE was found for mammals. In that fetal rat study, minor skeletal deformities were found, but no mention was made of the heart. The authors, however, referred to an abstract describing anatomical dissection of the rat embryo (8). This report mentioned, after decapitation, making a cut down each great artery with the heart beating, mainly to observe ventricular septal defects. This method would yield limited exposure of the perimembranous septum and the semilunar valves. Shunting of blood through a ventricular septal defect might be missed if the left ventricular pressure were low. It is possible that if the heart was examined, this methodology would have markedly restricted the ability to observe cardiac defects. Furthermore, a direct comparison may not be appropriate across species. We have recently published a study showing that DCE is also a cardiac teratogen in fetal rats (9).

In both the previous TCE study (6) and the present DCE study, the $25-\mu \mathrm{M}$ dose was not associated with increased cardiac abnormalities, whereas the $20-\mu \mathrm{M}$ dose produced the peak percentage of abnormalities. The reason for this is not clear. However, it is possible that the higher dose caused inactivation of hepatic enzyme systems that allow metabolism of the two compounds. This might cause the metabolites (trichloro- and dichloroacetyl aldehydes, ethanols, and acetic acids) to drop in concentration. Therefore, it seems possible that the metabolites were the actual teratogens, but data are not available to test this proposition.

In summary, this study shows that DCE exposure beginning on the $3 \mathrm{rd} \mathrm{d}$ of incubation produces a significant number of chick embryos with cardiac abnormalities and that this number is significantly greater than the number with other noncardiac abnormalities. Furthermore, DCE is at least as potent a cardiac teratogen as TCE, even though DCE was studied at lower doses. Data demonstrating that both TCE and DCE are cardiac teratogens are consistent with results of an epidemiologic study that demonstrated an association between drinking water contaminated with these two chemicals and the presence of human congenital cardiac disease.

\section{REFERENCES}

1. Condie LW 1985 Target organ toxicology of halocarbons commonly found contaminating drinking water. Sci Total Environ 47:433-441

2. Koskinen K, Hemminki K 1985 Experimental teratogenicity and embryotoxicity of occupational chemicals. In: Hemminki K, Sorsa M, Vainio H (eds) Occupational Hazards and Reproduction. Hemisphere Publishers, Washington, DC, pp 127-144

3. Murray FJ, Nitschke KD, Rampy LW, Schwetz BA 1979 Embryotoxicity and fetotoxicity of inhaled or ingested vinylidene chloride in rats and rabbits. Toxicol Appl Pharmacol 49:189-202

4. Swan SM, Deane M, Harris J, Neutra R 1985 Pregnancy outcomes in relation to water contamination, 1980-1981, San Jose, CA. In: Pregnancy Outcomes in Santa Clara County 1980-82: Reports of Two Epidemiological Studies. Epidemiological Studies Section, California Department of Health Services, Berkeley, CA

5. Goldberg SJ, Lebowitz MD, Graver E 1990 Association of high trichloroethylene levels in drinking water and increased incidence of congenital cardiac disease in children. J Am Coll Cardiol 16:155-164

6. Loeber CP, Diez de Pinos S, Goldberg SJ 1988 Trichloroethylene: a cardiac teratogen in developing chick embryos. Pediatr Res 24:740-744

7. de la Cruz MV, Munoz-Armas S, Munoz-Castellanos L 1972 Development of the Chick Heart. Johns Hopkins University Press, Baltimore, MD

8. Staples RE 1978 Detection of visceral abnormalities in mammalian fetuses. Teratology 17:48A(abstr)

9. Dawson BV, Johnson PD, Goldberg SJ, Ulreich JB 1990 Cardiac teratogenesis of trichloroethylene and dichloroethylene in a mammalian model. J Am Coll Cardiol 16:1304-1309* 